\title{
DISTRIBUIÇÃO DE N P K EM UM POVOAMENTO DE Ilex paraguariensis A. St.-Hil.
}

\author{
Gilvano Ebling Brondani*, Daniele Ukan**, Michele Fernanda Bortolini***, \\ Yorlene Chang Cambronero**, André Rossetto****, Carlos Bruno Reissmann***** \\ *Eng. Florestal, Mestrando em Engenharia Florestal, UFPR - gebrondani@yahoo.com.br \\ **Enga . Florestal, Mestranda em Engenharia Florestal, UFPR - daniukan@yahoo.com.br - yorchang@hotmail.com \\ ***Bióloga, Doutoranda em Produção Vegetal, UFPR - mibortolini@hotmail.com \\ ****Eng. Agrônomo, Mestrando em Ciências do Solo, UFPR - agrorossetto@yahoo.com.br \\ *****Eng. Florestal, Dr., Depto. de Solos e Engenharia Agrícola, UFPR - reissman@ufpr.br
}

Recebido para publicação: 17/05/2007 - Aceito para publicação: 24/08/2007

\begin{abstract}
Resumo
O presente trabalho teve como objetivo avaliar os teores nutricionais de nitrogênio $(\mathrm{N})$, fósforo $(\mathrm{P}) \mathrm{e}$ potássio (K) nas folhas, serapilheira e solo em um povoamento de 15 anos de idade de Ilex paraguariensis. $\mathrm{O}$ experimento foi conduzido no delineamento inteiramente casualizado, com a seleção de 15 árvores, classificadas de acordo com o porte e densidade de copa em três classes densa (I), média (II) e rala (III) -, em três grupos de cinco. Foi mensurada a altura total, a altura de copa, o diâmetro de copa e o peso seco de 100 folhas do terço médio. De cada árvore, coletaram-se quatro amostras de folhas do terço médio, quatro amostras de serapilheira e quatro amostras do solo na profundidade de $0-20 \mathrm{~cm}$, para análise química dos nutrientes. Os resultados obtidos indicaram que o teor de nitrogênio nas folhas é alto, os valores de fósforo são baixos e os de potássio apresentam valores considerados baixos em relação aos encontrados na literatura para a espécie. Não existiu diferença significativa entre os teores de macronutrientes nas classes de densidade de copa e solo, assim como do índice de recuo de $\mathrm{N}, \mathrm{P}$ e $\mathrm{K}$ das folhas da copa em relação à serapilheira.
\end{abstract}

Palavras-chave: Erva-mate; teor nutricional; acúmulo de macronutrientes.

\begin{abstract}
Distribution of N P K in a stand culture of Ilex paraguariensis A. St.-Hil.. The objective of this study was to evaluate nutritional levels of nitrogen $(\mathrm{N})$, phosphorus $(\mathrm{P})$ and potassium $(\mathrm{K})$ in leaves, litter and in the soil of a 15 years old stand of Ilex paraguariensis. The experiment was conducted in a completely randomized design. The 15 sample trees, were classified in three groups of five in accordance to their crown proportion and foliage density in classes: dense, medium and sparse. Total height, height and diameter of the crown were measured. In order to perform chemical analysis, leaves were sampled from four points of the middle of the crown and weighed to obtain the weight of 100 leaves. Four litter and soil samples were collected. The $\mathrm{N}$ content in leaves was high, the P was low and the $\mathrm{K}$, values were slightly below the reference data considered as low in the reported literature. The contents in the soil were considered high for $\mathrm{N}$ and low for $\mathrm{P}$ and $\mathrm{K}$. In relation to the crown classes no significant differences could be observed among the chemical data of the leaves, as for the soil. The nutrient retention index of the crown leaves in relation to litter leaves showed to be not significant.
\end{abstract}

Keywords: Erva-mate; nutritional level; macronutrient accumulation.

\section{INTRODUÇÃO}

Ilex paraguariensis A. St.-Hil., popularmente conhecida como erva-mate, pertencente à família Aquifoliaceae, é uma árvore de caule de cor acinzentada e que, quando cultivada, varia geralmente de 3 a $5 \mathrm{~m}$, porém na floresta pode alcançar $25 \mathrm{~m}$ de altura e $70 \mathrm{~cm}$ de DAP (diâmetro acima do peito) (CARVALHO, 2003). Essa espécie tem importância histórica na cultura e na economia da região Sul do Brasil e em países limítrofes, como Argentina, Uruguai e Paraguai (ZAMPIER, 2001). No Brasil, ocupa a Floresta Ombrófila Mista Montana (Floresta com Araucária) e a Floresta Estacional Semidecidual no 
noroeste do Paraná e no sul do Mato Grosso do Sul (MAZUCHOWSKI, 1989). Pode ser considerada como planta dióica, embora nas plantas femininas encontrem-se estames estéreis e nas plantas masculinas o pistilo que se deprime e aborta (BRAGAGNOLO et al., 1980).

Tradicionalmente, compõe um dos sistemas agroflorestais mais antigos e característicos da região Sul do Brasil, assumindo importância ambiental e socioeconômica significativa, além de ter sido, por um longo período, um dos primeiros produtos das exportações brasileiras (PENTEADO et al., 2000). O Brasil é o segundo maior produtor mundial de erva-mate, só perdendo para a Argentina, sendo o estado do Paraná o maior produtor nacional. A indústria nacional produz anualmente cerca de 270 mil toneladas de erva-mate, e o maior importador é o Uruguai, que absorve cerca de $81 \%$ da produção (PASINATO, 2004).

As inúmeras aplicações da erva-mate são decorrentes da composição química de suas folhas. Predomina a produção de bebidas, como o chimarrão (MACCARI JUNIOR e MAZUCHOWSKI, 2000). Nas últimas décadas, tem-se observado um crescimento e diversificação na sua linha de produtos, sendo apresentada aos consumidores a erva-mate triturada em diferentes tamanhos de partículas, associadas ou não à sacarose ou com plantas medicinais, e ainda inovando na apresentação desses produtos - da tradicional embalagem de papel para a embalagem plástica (DONADUZZI et al., 2003).

A produtividade de um povoamento florestal pode ser estimada pelo conhecimento da sua dinâmica de nutrientes, já que existe uma relação direta entre teores de nutrientes no solo e nas folhas e a produtividade da planta (GALLO, 1972). Ao se conhecer a fertilidade do solo e as condições nutricionais das árvores, é possível estabelecer estratégias de manejo nutricional para a obtenção de uma produção florestal sustentável (GARRIDO, 1988; LEITE et al., 1998).

Quando se trata da composição da serapilheira em um povoamento florestal, não se deve levar em conta apenas a quantidade de matéria orgânica devolvida ao solo, mas também sua qualidade, que é representada pelo teor dos elementos químicos nela existentes (GARRIDO, 1988).

O nitrogênio é um dos elementos minerais requeridos em maior quantidade pelas plantas, e o que mais limita o crescimento. Ele faz parte de proteínas, ácidos nucléicos, amidas, coenzimas e muitos outros importantes constituintes celulares, incluindo membranas e diversos hormônios vegetais (SOUZA; FERNANDES, 2006; TAIZ; ZEIGER, 2004).

O fósforo é um componente integral de compostos importantes das células vegetais, incluindo fosfato-açúcares, intermediário da respiração e fotossíntese, bem como os fosfolipídeos, que compõem as membranas vegetais (TAIZ; ZEIGER, 2004). Além disso, ele está relacionado com aquisição, estocagem e utilização de energia (EPSTEIN; BLOOM, 2004).

Já o potássio, presente nas plantas como $\mathrm{K}^{+}$, desempenha um importante papel na regulação do potencial osmótico das células vegetais (TAIZ; ZEIGER, 2004). Embora seja o mais abundante mineral catiônico presente nas plantas e possa constituir até $10 \%$ do peso seco, não é um constituinte integral de qualquer metabólico que pode ser isolado de material vegetal (EPSTEIN; BLOOM, 2004). Segundo Meurer (2006), esse elemento tem importante função no estado energético da planta, na translocação e armazenamento de assimilados e na manutenção da água nos tecidos vegetais.

Sendo assim, este trabalho teve como objetivo avaliar os teores nutricionais de $\mathrm{N}, \mathrm{P}$ e $\mathrm{K}$ nas folhas, na serapilheira e no solo em um povoamento de Ilex paraguariensis A. St.-Hil. aos 15 anos de idade, estabelecido no município de Pinhais (PR).

\section{MATERIAL E MÉTODOS}

O experimento foi realizado no ano de 2006, na área da Estação Experimental do Canguiri, da Universidade Federal do Paraná, município de Pinhais (PR). O solo local foi classificado como Cambissolo álico A proeminente textura argilosa, relevo ondulado, substrato argilito da formação Guabirotuba (WISNIEWSKI et al., 1996). Segundo Maack (2002), o clima é classificado como Cfb, ou seja, temperatura do mês mais frio entre $18 \mathrm{e}-3{ }^{\circ} \mathrm{C}$, sempre úmido, chuva em todos os meses do ano e temperatura do mês mais quente menor que $22^{\circ} \mathrm{C}$, mas no mínimo quatro meses com temperatura maior que $10^{\circ} \mathrm{C}$.

As árvores utilizadas no experimento foram implantadas em 1991, no delineamento blocos completos ao acaso, com cinco blocos, sendo cada bloco formado por três parcelas, cada parcela com 60 árvores, espaçadas de $3 \mathrm{~m}$ na linha e $3 \mathrm{~m}$ na entrelinha (WISNIEWSKI et al., 1996). Para a realização do presente estudo, em maio de 2006, desconsiderou-se o delineamento em blocos da fase de implantação do povoamento, sendo selecionadas 15 árvores ao acaso e classificadas visivelmente de acordo com seu 
porte e densidade de copa: densa (I), média (II) e rala (III), em três grupos de cinco, segundo delineamento inteiramente casualizado. Posteriormente, foi realizada a medição da altura total, da altura de copa e do diâmetro de copa das respectivas árvores.

De cada árvore selecionada, foram coletadas 4 amostras de folhas do terço médio e 4 amostras de serapilheira na disposição norte-sul e leste-oeste, formando uma amostra composta de cada compartimento. Da mesma forma, foi coletado o solo de 0-20 cm, com tradagem, na periferia da projeção da copa de cada árvore.

As medições foram realizadas utilizando-se fita métrica e iniciando-se pela face norte, assim como a retirada das amostras, com auxílio de uma bússola. Todas as árvores foram enumeradas e etiquetadas, as amostras de solo foram acondicionadas em sacos plásticos e as folhas e serapilheira em sacos de papel. Em seguida, essas amostras foram encaminhadas para o laboratório e submetidas à análise química, para a determinação de nutrientes e para a determinação da massa seca de 100 folhas (FIEDLER et al., 1973; BORILLE, 2004). Para tanto, as amostras foliares foram secas em estufa a $60{ }^{\circ} \mathrm{C}$ até peso constante, e, para análise química, as folhas sem os pecíolos foram moídas até a consistência de pó.

Para a análise foliar e da serapilheira, o procedimento analítico utilizado em laboratório na determinação do nitrogênio total foi o Semi-micro Kjeldahl (BREMNER, 1996). Já na determinação de P e K, utilizou-se o método de digestão via seca (PERKIN-ELMER, 1973; JONES; CASE, 1990), com posterior determinação por colorimetria e fotometria de chama, respectivamente.

Nesse estudo, visando determinar a relação do teor de nutriente que fica retido nas folhas da copa da planta com o teor de nutriente que é exportado para serapilheira, calculou-se a diferença do teor nutricional entre esses dois compartimentos, denominando essa variável de índice de recuo (IR). O IR foi determinado da seguinte maneira:

$$
I R=T N F-T N S
$$

Onde: $\mathrm{IR}=$ índice de recuo, $\mathrm{TNF}=$ teor de nutriente na folha da copa e TNS = teor de nutriente na folha da serapilheira.

O experimento foi conduzido em delineamento inteiramente casualizado, com três classes de árvores - densa (I), média (II) e rala (III) - e cinco repetições, totalizando 15 árvores. Os dados foram submetidos à análise de variância por meio de teste $\mathrm{F}(\mathrm{P}<0,05)$, tendo sido as médias discriminadas pelo teste de Tukey a 5\% de probabilidade de erro.

\section{RESULTADOS E DISCUSSÃO}

Com base na análise de variância das características amostradas, pode-se observar que existe efeito significativo da altura total, da altura da copa, do diâmetro de copa médio e do peso seco de 100 folhas em relação às classes das árvores (Tabela 1 ).

Tabela 1. Resultados da análise de variância para altura total, altura de copa, diâmetro de copa médio (DCM) e peso seco de 100 folhas (PF) por classe de copa para um povoamento de Ilex paraguariensis A. St.-Hil.

Table 1. Variance analysis results of the total height, crown height, diameter of the crown (DCM) and dry weight of 100 leaves (PF) for crown classes of a stand of Ilex paraguariensis A. St.-Hil.

\begin{tabular}{|c|c|c|c|c|c|}
\hline \multirow{2}{*}{ Causas da variação } & \multirow{2}{*}{ GL } & \multicolumn{4}{|c|}{ Quadrados médios } \\
\hline & & Altura total (m) & Altura da copa (m) & DCM (m) & PF (g) \\
\hline Classe & 2 & $1,5120^{* *}$ & $1,3020^{* *}$ & $0,6476^{* *}$ & $405,96 *$ \\
\hline Resíduo & 12 & 0,0797 & 0,0527 & 0,0803 & 100,17 \\
\hline Média & - & 3,10 & 2,46 & 2,16 & 39,55 \\
\hline CV (\%) & - & 9,10 & 9,33 & 13,09 & 25,31 \\
\hline
\end{tabular}

Analisando-se as variáveis mensuradas das árvores de Ilex paraguariensis A. St.-Hil. (Tabela 2), verifica-se que, para altura total e diâmetro médio da copa, a classe III apresentou a menor média, 2,50 m e 1,77 m, respectivamente, diferindo estatisticamente das demais. Já para a altura da copa, a proporção foi conforme a classificação das árvores, sendo a classe I a que apresentou a maior média de altura da copa. Para o peso seco das 100 folhas, a classe I diferiu estatisticamente da classe II, com 46,69 g. 
Tabela 2. Valores médios das características da altura total, da altura de copa, do diâmetro de copa médio (DCM) e do peso seco de 100 folhas (PF), por classe de copa, para um povoamento de Ilex paraguariensis A. St.-Hil.

Table 2. Average values of the characteristics of the total height, crown height, diameter of the crown (DCM) and dry weight of 100 leaves (PF) for crown classes of a stand of Ilex paraguariensis A. St.-Hil.

\begin{tabular}{lcccc}
\hline Classe & $\begin{array}{c}\text { Altura total }(\mathbf{m}) \\
(\mathbf{m e ́ d i a} \pm \boldsymbol{\sigma})\end{array}$ & $\begin{array}{c}\text { Altura da copa } \mathbf{( m )} \\
(\mathbf{m e ́ d i a} \pm \boldsymbol{\sigma})\end{array}$ & $\begin{array}{c}\text { DCM }(\mathbf{m}) \\
(\mathbf{m e ́ d i a} \pm \boldsymbol{\sigma})\end{array}$ & $\begin{array}{c}\text { PF }(\mathbf{g}) \\
(\mathbf{m e ́ d i a} \pm \boldsymbol{\sigma})\end{array}$ \\
\hline I & $3,58^{\mathrm{a}} \pm 0,18$ & $2,96 \mathrm{a} \pm 0,09$ & $2,47 \mathrm{a} \pm 0,38$ & $46,69 \mathrm{a} \pm 12,90$ \\
II & $3,22 \mathrm{a} \pm 0,41$ & $2,48 \mathrm{~b} \pm 0,37$ & $2,26 \mathrm{a} \pm 0,26$ & $29,42 \mathrm{~b} \pm 3,30$ \\
III & $2,50 \mathrm{~b} \pm 0,20$ & $1,94 \mathrm{c} \pm 0,11$ & $1,77 \mathrm{~b} \pm 0,17$ & $42,53 \mathrm{ab} \pm 11,10$ \\
\hline
\end{tabular}

Médias seguidas da mesma letra não diferem estatisticamente pelo teste de Tukey ao nível de $5 \%$ de probabilidade de erro. $\sigma=$ desvio padrão da média.

Quanto à análise química, não foi encontrada correlação entre os macronutrientes do solo em relação à folha e à serapilheira e nem da folha em relação à serapilheira, nas diferentes classes amostradas.

Em relação ao teor de N, P e K, não existiu interação entre a classe de árvores com a posição (folha e serapilheira), assim como as classes não diferiram quanto à distribuição desses nutrientes (Tabela 3). Entretanto, existe efeito significativo quanto à distribuição desses nutrientes em relação à posição, ou seja, os nutrientes N, P e K diferiram quanto ao teor na folha e serapilheira independentemente da classe.

Tabela 3. Resultados da análise de variância para o teor de $\mathrm{N}, \mathrm{P}$ e $\mathrm{K}$, em $\mathrm{g} \mathrm{kg}^{-1}$, na folha e serapilheira por classe, para um povoamento de Ilex paraguariensis A. St.-Hil.

Table 3. Variance analysis results for the nutritional levels of $\mathrm{N}, \mathrm{P}$ and $\mathrm{K}, \mathrm{g} \mathrm{kg}^{-1}$, in the leaves and litter for crown classes of a stand of Ilex paraguariensis A. St.-Hil.

\begin{tabular}{|c|c|c|c|c|}
\hline \multirow{2}{*}{ Causas da variação } & \multirow{2}{*}{ GL } & \multicolumn{3}{|c|}{ Quadrados médios } \\
\hline & & $\mathbf{N}^{(1)}$ & $\mathbf{P}^{(1)}$ & $\mathbf{K}$ \\
\hline Classe & 2 & $0,0116^{\mathrm{ns}}$ & $0,00004^{\mathrm{ns}}$ & $27,64^{\mathrm{ns}}$ \\
\hline Posição & 1 & $8,0235 * *$ & $0,13520 * *$ & $576,03 * *$ \\
\hline Classe * Posição & 2 & $0,0420^{\mathrm{ns}}$ & $0,00049^{\mathrm{ns}}$ & $1,90^{\mathrm{ns}}$ \\
\hline Resíduo & 24 & 0,0327 & 0,00027 & 30,62 \\
\hline Média & - & 29,74 & 0,53 & 11,41 \\
\hline C.V. $(\%)$ & - & 11,06 & 7,45 & 48,49 \\
\hline
\end{tabular}

Comparando-se as médias das concentrações de macronutrientes das folhas verdes com as coletadas na serapilheira, observa-se uma diminuição nos teores de N, P e K nas folhas desta (Figura 1). Isso acontece porque esses elementos são altamente móveis dentro da planta, concentrando-se principalmente nos sítios de maior atividade, como nas folhas remanescentes na copa (CALDEIRA et al., 2000), ou para tecidos de reserva (TAIZ; ZEIGER, 2004).

O povoamento apresenta altos teores de nitrogênio foliar (Figura 1a), possivelmente resultado do bom conteúdo de matéria orgânica no solo (Tabela 4). Radomski et al. (1992) consideram valores maiores que $23 \mathrm{~g} \mathrm{~kg}^{-1}$ de $\mathrm{N}$ como adequados para erva-mate. Por outro lado, a presença de espécies leguminosas consorciadas, como neste caso o timbó (Ateleia glazioviana), pode estar favorecendo esse elevado conteúdo de nitrogênio no solo. A inserção de espécies fixadoras de N pode ser interessante no manejo de culturas florestais, pois a introdução desse elemento, por meio da serapilheira, pode também aumentar o estoque de nutrientes para os cultivos em sucessão ou rotação (BALIEIRO et al., 2004). Sendo assim, seriam importantes estudos que comprovassem a relação do timbó (Ateleia glazioviana) com a erva-mate nessa área. Reissmann et al. (1985) observaram, em um estudo de variação anual de $\mathrm{N}, \mathrm{P}$ e $\mathrm{K}$ em folhas de erva-mate, que o $\mathrm{N}$ se mantém relativamente estável ao longo do ano, com um pico máximo em outubro e diminuindo sensivelmente até abril. 


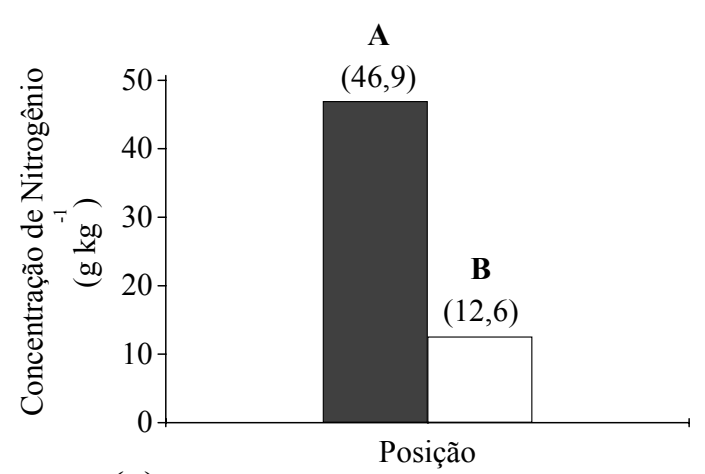

(a)

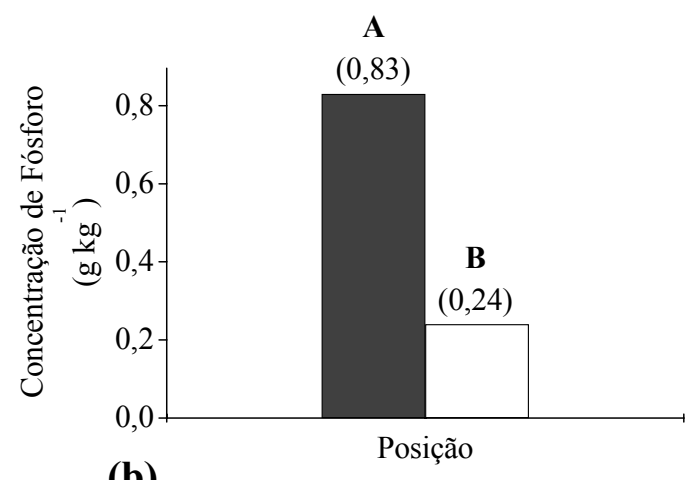

(b)

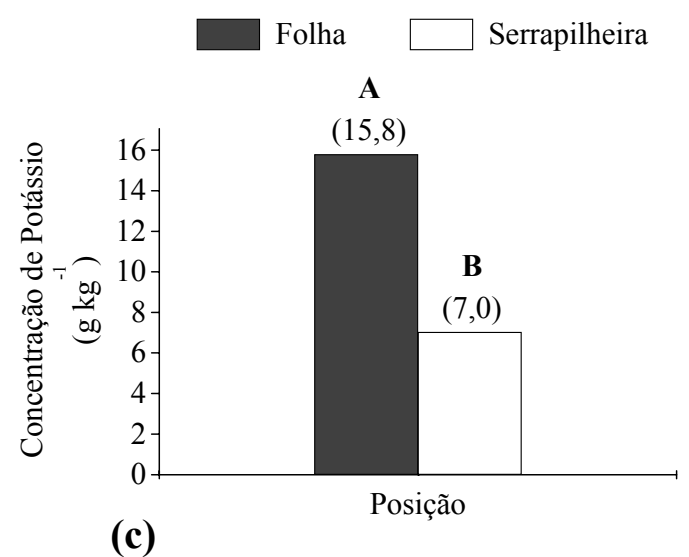

Figura 1. Valores médios das concentrações de nitrogênio (a), fósforo (b) e potássio (c) das folhas e serapilheira do povoamento de Ilex paraguariensis A. St.-Hil. Médias seguidas por uma mesma letra não diferem estatisticamente pelo teste de Tukey a $5 \%$ de probabilidade.

Figure 1. Nutricional level of nitrogen (a), phosphorus (b) and potassium (c) in the leaves and litter of a stand of Ilex paraguariensis A. St.-Hil. Averages followed by the same letter do not differ by the Tukey test, at $5 \%$ probability.

Tabela 4. Resultados da análise de variância para o índice de recuo, entre as folhas e a serapilheira, de N, $\mathrm{P}$ e K $\left(\mathrm{g} \mathrm{kg}^{-1}\right)$ das classes de copa de um povoamento de Ilex paraguariensis A. St.-Hil.

Table 4. Variance analysis results for the nutrient retention index between the leaves and litter of $\mathrm{N}, \mathrm{P}$ and $\mathrm{K}\left(\mathrm{g} \mathrm{kg}^{-1}\right)$ of the crown classes of a stand of Ilex paraguariensis A. St.-Hil.

\begin{tabular}{lcccc}
\hline \multirow{2}{*}{ Causas da variação } & \multirow{2}{*}{ GL } & $\mathbf{N}$ & $\mathbf{P}$ & Quadrados médios \\
\cline { 3 - 5 } & & $104,90^{\mathrm{ns}}$ & $0,0203^{\mathrm{ns}}$ & $3,81^{\text {ns }}$ \\
Classe & 2 & 103,72 & 0,0110 & 85,74 \\
Resíduo & 12 & 34,37 & 0,59 & 8,76 \\
Média & - & 29,64 & 17,88 & 105,66 \\
C.V. (\%) & - & - &
\end{tabular}

Por outro lado, o povoamento apresenta baixos teores de P nas folhas (Figura 1b). Sosa (1992) considera baixos os valores de fósforo entre 0,8 e $2,8 \mathrm{~g} \mathrm{~kg}^{-1}$. Reissmann et al. (1985) citam essa condição como característica nutricional da espécie, e não um caso de deficiência, já que não foram observados sintomas de deficiência entre as erveiras. Da revisão de Oliveira e Rotta (1985), pode-se depreender que isso deve estar associado a áreas de distribuição natural da planta em solos ácidos, dificultando a absorção desse nutriente. 
Outra explicação para os baixos teores de $\mathrm{P}$ nas folhas deste povoamento seria o fato de que, segundo Reissmann et al. (1985), para erva-mate, existe uma coincidência entre o ritmo fenológico e a variação do nível de nutrientes na copa. A coleta do material para essas análises foi realizada em março de 2006, mês que corresponde, segundo Carvalho (2003), à época de maturação dos frutos no estado do Paraná, que atuariam como dreno desse nutriente, diminuindo seu conteúdo nas folhas (SALISBURY; ROSS, 1992). Reissmann et al. (1985), no seu estudo de variação anual, encontraram valores mínimos de $\mathrm{P}$ em fevereiro.

Os teores de K apresentaram-se acima (Figura 1c) dos valores que Sosa (1992) considera baixos para erva-mate, ou seja, na faixa de 12,2 a 14,9 $\mathrm{g} \mathrm{kg}^{-1}$. A elevada concentração de $\mathrm{Mg}$ no solo (Tabela 6) pode estar inibindo o K no solo, limitando sua disponibilização para as plantas, pela relação antagônica entre o $\mathrm{K}$ e o $\mathrm{Mg}$ (TISDALE et al., 1993).

$\mathrm{O}$ índice de recuo de $\mathrm{N}, \mathrm{P}$ e $\mathrm{K}$ entre as folhas e a serapilheira não apresentou diferença significativa em relação às diferentes classes (Tabela 4).

Avaliando o índice de recuo dos nutrientes, foi possível verificar que, para o N, o valor do recuo foi grande em relação ao que se perde na serapilheira (Tabela 5). Isso se deve à necessidade da planta de altas concentrações de N para a produção da biomassa (KERBAUY, 2004).

Tabela 5. Índice de recuo dos nutrientes das folhas em relação à serapilheira no povoamento de Ilex paraguariensis A. St.-Hil.

Table 5. Nutrient retention index of the leaves in relation to the litter of a stand of Ilex paraguariensis A. St.-Hil.

\begin{tabular}{|c|c|c|c|}
\hline \multirow[b]{2}{*}{ Classe } & \multicolumn{3}{|c|}{ Índice de recuo } \\
\hline & $\begin{array}{c}N \\
\left(\mathrm{~g} \mathrm{~kg}^{-1}\right)(\mathrm{média} \pm \sigma)\end{array}$ & $\begin{array}{c}P \\
\left(\mathrm{~g} \mathrm{~kg}^{-1}\right)(\text { média } \pm \sigma)\end{array}$ & $\begin{array}{c}\mathrm{K} \\
\left(\mathrm{g} \mathrm{kg}^{-1}\right)(\mathrm{média} \pm \sigma)\end{array}$ \\
\hline 1 & $29,16 \pm 12,48$ & $0,53 \pm 0,14$ & $9,72 \pm 13,95$ \\
\hline 2 & $36,18 \pm 4,81$ & $0,66 \pm 0,03$ & $8,55 \pm 3,08$ \\
\hline 3 & $37,76 \pm 11,50$ & $0,57 \pm 0,11$ & $8,02 \pm 7,30$ \\
\hline Média & 34,37 & 0,59 & 8,76 \\
\hline
\end{tabular}

$\sigma=$ desvio padrão da média.

Tabela 6. Análise química do solo quanto à divisão das classes de copas do povoamento de Ilex paraguariensis A. St.-Hil.

Table 6. Chemical analysis of the soil for the tree crown classes of the a stand of Ilex paraguariensis A. St.-Hil.

\begin{tabular}{|c|c|c|c|c|}
\hline \multirow[b]{2}{*}{ Atributo } & \multicolumn{3}{|c|}{ Classe } & \multirow{2}{*}{$\begin{array}{c}\text { Média } \\
(\operatorname{média} \pm \sigma)\end{array}$} \\
\hline & $\begin{array}{c}1 \\
(\text { média } \pm \sigma)\end{array}$ & $\begin{array}{c}2 \\
(\text { média } \pm \sigma)\end{array}$ & $\begin{array}{c}3 \\
(\text { média } \pm \sigma)\end{array}$ & \\
\hline $\mathrm{pH} \mathrm{CaCl}{ }_{2}$ & $4,6 \pm 0,18$ & $4,5 \pm 0,18$ & $4,6 \pm 0,26$ & $4,6 \pm 0,20$ \\
\hline SMP & $5,0 \pm 0,27$ & $5,0 \pm 0,28$ & $5,1 \pm 0,37$ & $5,0 \pm 0,29$ \\
\hline $\mathrm{Al}^{3+}\left(\mathrm{cmol}_{\mathrm{c}} \mathrm{dm}^{-3}\right)$ & $1,6 \pm 1,02$ & $1,9 \pm 0,79$ & $1,3 \pm 0,92$ & $1,6 \pm 0,89$ \\
\hline $\mathrm{H}^{+}+\mathrm{Al}^{3+}\left(\mathrm{cmol}_{\mathrm{c}} \mathrm{dm}^{-3}\right)$ & $10,3 \pm 2,05$ & $10,8 \pm 2,28$ & $10,0 \pm 2,80$ & $10,4 \pm 2,24$ \\
\hline $\mathrm{Ca}^{2+}\left(\mathrm{cmol}_{\mathrm{c}} \mathrm{dm}^{-3}\right)$ & $4,5 \pm 1,14$ & $3,8 \pm 0,82$ & $4,8 \pm 1,21$ & $4,4 \pm 1,09$ \\
\hline $\mathrm{Mg}^{2+}\left(\mathrm{cmol}_{\mathrm{c}} \mathrm{dm}^{-3}\right)$ & $3,0 \pm 0,42$ & $2,3 \pm 0,36$ & $3,0 \pm 0,61$ & $2,8 \pm 0,55$ \\
\hline $\mathrm{K}^{+}\left(\mathrm{cmol}_{\mathrm{c}} \mathrm{dm}^{-3}\right)$ & $0,086 \pm 0,015$ & $0,106 \pm 0,017$ & $0,088 \pm 0,047$ & $0,093 \pm 0,029$ \\
\hline $\mathrm{SB}\left(\mathrm{cmol}_{\mathrm{c}} \mathrm{dm}^{-3}\right)$ & $7,61 \pm 1,50$ & $6,21 \pm 0,99$ & $7,89 \pm 1,69$ & $7,23 \pm 1,52$ \\
\hline $\mathrm{T}\left(\mathrm{cmol}_{\mathrm{c}} \mathrm{dm}^{-3}\right)$ & $17,9 \pm 0,74$ & $17,0 \pm 1,95$ & $17,9 \pm 1,64$ & $17,6 \pm 1,49$ \\
\hline $\mathrm{P}\left(\mathrm{mg} \mathrm{dm}^{-3}\right)$ & $2,3 \pm 0,48$ & $2,1 \pm 0,38$ & $1,9 \pm 0,31$ & $2,1 \pm 0,40$ \\
\hline $\mathrm{C}\left(\mathrm{g} \mathrm{dm}^{-3}\right)$ & $45,4 \pm 2,99$ & $43,7 \pm 2,63$ & $42,2 \pm 2,93$ & $43,8 \pm 2,95$ \\
\hline $\mathrm{V}(\%)$ & $42,6 \pm 9,26$ & $36,8 \pm 7,19$ & $44,8 \pm 11,48$ & $41,4 \pm 9,44$ \\
\hline$M(\%)$ & $17,4 \pm 12,28$ & $23,4 \pm 9,40$ & $14,8 \pm 11,52$ & $18,5 \pm 10,96$ \\
\hline $\mathrm{Ca} / \mathrm{Mg}$ & $1,5 \pm 0,23$ & $1,6 \pm 0,35$ & $1,6 \pm 0,25$ & $1,6 \pm 0,28$ \\
\hline Argila $\left(\mathrm{g} \mathrm{kg}^{-1}\right)$ & $595,0 \pm 27,39$ & $577,5 \pm 41,83$ & $575,0 \pm 17,68$ & $582,5 \pm 29,81$ \\
\hline
\end{tabular}


Conforme CQFS-RS/SC (2004), o solo dessa área (Tabela 6) foi classificado com teores muito baixos de $\mathrm{P}$, entretanto o índice de recuo foi alto (Tabela 5), ou seja, existe uma grande retenção do $\mathrm{P}$ pela planta antes da perda da folha, já que a disponibilidade no solo desse nutriente é baixa. Já para o índice de recuo de $\mathrm{K}$, foram constatados índices menores (Tabela 5), sendo maior a perda de $\mathrm{K}$ para a serapilheira.

\section{CONCLUSÕES}

Nas condições em que foi desenvolvido este trabalho, conclui-se que, para erva-mate aos 15 anos de idade, o teor de nitrogênio nas folhas é alto, os valores de fósforo são baixos e os de potássio apresentam-se sensivelmente acima dos valores considerados baixos na literatura pertinente. Quanto ao solo, o teor de nitrogênio encontrado foi alto, o fósforo apresentou valores muito baixos e o potássio valores baixos.

Não houve diferença significativa entre os teores de macronutrientes nas classes de densidade de copa, podendo-se considerar neste caso que o desenvolvimento heterogêneo das árvores nas suas características dasométricas tem maior relação com o material genético plantado do que com o estado nutricional do povoamento para os elementos investigados.

\section{REFERÊNCIAS}

BALIEIRO, F. C. de.; FRANCO, A. A.; PEREIRA, M. G.; CAMPELLO, E. F. C.; DIAS, L. E.; FARIA, S. M. de.; ALVES, B. J. M. Dinâmica da serapilheira e transferência de nitrogênio ao solo, em plantios de Pseudosamanea guachapele e Eucalyptus grandis. Pesquisa Agropecuária Brasileira, Brasília, v. 39, n. 6, p. 597-601, 2004.

BORILLE, A. W. Relação entre compostos fotoquímicos e o nitrogênio em morfotipos da erva-mate (Ilex paraguariensis St. Hil.). 109 f. Dissertação (Mestrado em Engenharia Florestal) - Curso de PósGraduação em Engenharia Florestal da Universidade Federal do Paraná, Curitiba, 2004.

BRAGAGNOLO, N.; PAN, W.; FILHO, L. K. Manual técnico de erva-mate. Curitiba, PR, 1980. 40 p.

BREMNER, J. M. Nitrogen-Total. In: SPARKS, D. L. Methods of Soil Analysis. Part 3 chemical methods. Madison: SSSA Book Series, 1996. p. 1085-1121.

CALDEIRA, M. V. W.; SCHUMACHER, M. V.; TEDESCO, N.; SANTOS, E. M. dos. Ciclagem de nutrientes em Acacia mearnsii de Wild. V. Quantificação do conteúdo de nutrientes na biomassa aérea de Acacia mearnsii de Wild. Procedência australiana. Ciência Rural, Santa Maria, v. 30, n. 6, p. 977-982, 2000.

CARVALHO, P. E. R. Espécies Arbóreas Brasileiras. Embrapa, Informação Tecnológica, Colombo: Embrapa Florestas, 2003. 1039p.

CQFS-RS/SC - Comissão de Química e Fertilidade do Solo. Manual de adubação e calagem para os estados do Rio Grande do Sul e de Santa Catarina. Ed. 10. Porto Alegre: Evangraf LTDA, SBCSNúcleo Regional Sul, 2004. 400 p.

DONADUZZI, C. M.; CARDOZO JÚNIOR, E. L.; DONADUZZI, E. M.; MANFIO, J. L. Avaliação da presença de contaminantes microbiológicos em amostras de erva-mate (Ilex paraguariensis St. Hil.) comercializadas em embalagens de papel e laminados. In: CONGRESSO SUL-AMERICANO DA ERVA-MATE, 3., Feira do Agronegócio da Erva-Mate, 1., 2003, Chapecó, Anais... 2003. 1 CD-ROM.

EPSTEIN, E.; BLOOM, A. Nutrição mineral de plantas: princípios e perspectivas. Tradução: Maria Edna Tenório Nunes. Ed. 2. Londrina: Editora Planta, 2004. 403 p.

FIEDLER, H. J.; NEBE, W.; HOFFMANN, F. Forstlichepflanzenernährung und Düngung. Stuttgart: Gustav Fischer Verlag, 1973. 481 p.

GALLO, J. R. Análise foliar como meio de caracterização de propriedades químicas do solo. In: MONIZ, A. C. Elementos de pedologia. São Paulo: Polígono, 1972. p. 251-256. 
GARRIDO, M. A. de. O. Caracteres silviculturais e conteúdo de nutrientes no folhedo de alguns povoamentos puros e misto de espécies nativas. $105 \mathrm{f}$. Tese (Doutorado em Ciências) - Escola Superior de Agricultura Luiz de Queiroz, São Paulo, 1988.

JONES, Jr. J. B.; CASE, V. W. Sampling handling and analyzing plant tissue samples. In:

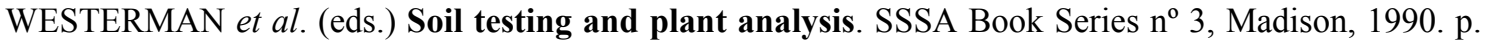
$389-427$

KERBAUY, G. B. Fisiologia Vegetal. Rio de janeiro: Guanabara Koogan, 2004. 452p.

LEITE, F. P.; BARROS, N. F.; NOVAIS, R. F.; FABRES, A. S. Acúmulo e distribuição de nutrientes em Eucalyptus grandis sob diferentes densidades populacionais. Revista Brasileira de Ciência do Solo, v. 22, p. 419-426, 1998.

MAACK, R. Geografia física do estado do Paraná. Ed. 3. Curitiba: Imprensa Oficial, 2002. 440 p.

MACCARI JUNIOR, A.; MAZUCHOWSKI, J. Z. Produtos alternativos e desenvolvimento da tecnologia industrial na cadeia produtiva da erva-mate. Câmara Setorial da Cadeia Produtiva da Erva-Mate, Paraná MCT/CNPq/PROJETO PADCT Erva-Mate, Curitiba, 2000. 176p.

MAZUCHOWSKI, J. Z. Princípios metodológicos para geração e difusão de tecnologia florestal. Convênio FAO - Paraná - França, Projeto Bracatinga, EMATER - Paraná, Curitiba, 1989. 68 p.

MEURER, E. J. Potássio. In: FERNANDES, M. S. Nutrição mineral de plantas. Minas Gerais: Sociedade Brasileira de Ciência do Solo, Viçosa, 2006. p. 281-298.

OLIVEIRA, Y. M. M.; ROTTA, E. Área de distribuição natural da erva-mate. In: SEMINARIO SOBRE ATUALIDADES E PERSPECTIVAS FLORESTAIS: SILVICULTURA DA ERVA-MATE, 10., 1985, Curitiba. Anais... Curitiba: Embrapa - Centro Nacional de Pesquisa de Florestas, 1985. p. 17-36.

PASINATO, R. Aspectos etnoentomológicos, sócioeconômicos e ecológicos relacionados à cultura da erva-mate (Ilex paraguariensis) no município de Salto do Lontra, Paraná, Brasil. 112 f. Dissertação (Mestrado) - Escola Superior de Agricultura Luiz de Queiroz, São Paulo, 2004.

PENTEADO, S. R. C.; IEDE, E. T.; LEITE, M. S. P. Pragas da erva-mate: perspectivas de controle. In: CONGRESSO SUL-AMERICANO DA ERVA-MATE, 2.; REUNIÃO TÉCNICA DA ERVA-MATE, 3., 2000, Porto Alegre. Anais... Porto Alegre: Ed. UFRGS, 2000. p. 27-34.

PERKIN-ELMER. Analytical Methods of Atomic Absorption Spectrophotometry. Analytical methods agriculture. Section AY-II. Analysis of Feeds-Norwalk: Perkin-Elmer Corporation, 1973. 476p.

RADOMSKI, R. M. B.; REISSMANN, C. B.; QUADROS, R. M. B. Comparação dos teores de macro e micronutrientes em folhas de erva-mate (Ilex paraguariensis St. Hil.) a céu aberto. In: REUNIÃO TÉCNICA DO CONE SUL SOBRE A CULTURA DA ERVA-MATE, 1992, Porto Alegre. Programas e Resumos... Porto Alegre: FAPERGS, 1992. p. 30.

REISSMANN, C. B.; KOEHLER, C.; DA ROCHA, H. O.; HILDEBRAND, E. E. Avaliação das exportações de macronutrientes pela exploração da erva-mate. In: SEMINARIO SOBRE ATUALIDADES E PERSPECTIVAS FLORESTAIS: SILVICULTURA DA ERVA-MATE, 10., 1985, Curitiba. Anais... Curitiba: Embrapa - Centro Nacional de Pesquisa de Florestas, 1985. p. 128-140.

SALISBURY, F. B.; ROSS, C. W. Plant physiology. $4^{\text {th }}$ edition. Belmont: Wadsworth Publishing Company, 1992. $682 \mathrm{p}$.

SOSA, D. A. Evaluación de la productividad del cultivo de yerba mate em relación al estado nutricional suelo/planta. In: CURSO DE CAPACITACIÓN EN PRODUCCIÓN DE YERBA MATE, 1., 1992, Cerro Azul. Anais... Cerro Azul: INTA - Estación Experimental Agropecuaria Cerro Azul, 1992. p. 61-64.

SOUZA, S. R.; FERNANDES, M. S. Nitrogênio. In: FERNANDES, M. S. Nutrição mineral de plantas. Viçosa, Minas Gerais: Sociedade Brasileira de Ciência do Solo, 2006. p. 215-252. 
TAIZ, L.; ZEIGER, E. Fisiologia vegetal. Tradução: SANTARÉM, E. R. [et al.]. Ed. 3. Porto Alegre: Artmed, 2004. 719 p.

TISDALE, S. L.; NELSON, W. L.; BEATON, J. D.; HAVLIN, J. L. Soil fertility and fertilizers. $5^{\text {th }}$ edition. New York: MacMillan Publishing Company, 1993. 634 p.

WISNIEWSKI, C. et al. Exportação de biomassa e macronutrientes com a primeira poda de formação da erva-mate na região de Pinhais - PR. Editora: UFPR, Agrárias, Curitiba, v.15, n. 2, 1996. p.179-186.

ZAMPIER, A. C. Avaliação dos níveis de nutrientes, cafeína e taninos após adubação mineral e orgânica, e sua relação com a produtividade na erva-mate (Ilex paraguariensis St. Hil.). $103 \mathrm{f}$. Dissertação (Mestrado em Silvicultura) - Universidade Federal do Paraná, Curitiba, 2001. 